\title{
Detecting Abnormal Activities using Computer Vision in Big Data Framework
}

\author{
Richa Gupta, Amit Gupta, Vikas Tripathi, Devesh Pratap Singh, Bhaskar Pant
}

\begin{abstract}
The abnormal behaviour of any person can be detected using computer vision. This is an important area in the field of research which is driven by wide variety of domains like intelligent video surveillance. Various techniques can be used in the field of computer vision feature extraction and description scheme. In this paper we have shown the comparison of all the techniques and method used in computer vision for the detection of abnormal activities.
\end{abstract}

Keywords: Object detection, abnormal activities detection, Event detection, Big data and Computer vision.

\section{INTRODUCTION}

This paper is a systematic review of object detection, abnormal activities detection, event detection, object behaviour detection, video summarization and synopsis generation. It shows a comparative study of various techniques used for the detection of any kind of abnormal activity. It provides a detailed comparison of different technologies so that a researcher can select a specific tool and technique for its work in the field of computer vision or detection of abnormal activities. This study is further sub divided into the following sections: Big Data and Computer Vision which describes various fundamental techniques of Computer Vision and some concepts of Big Data Analytics. The next section describes the various techniques used in Computer Vision. Next is the Literature Review section which specifies the detailed description of all the techniques used in this area and associated research work done in this field. Finally the Conclusion is given which displays the comparison of different techniques with their applications.

\section{BIG DATA AND COMPUTER VISION}

The term Computer Vision can be described as the "Techniques or Technologies that provides the information acquiring facility to computer". It is basically interdisciplinary scientific terminology that automates the tasks which can be easily done by human visual system. The main usage of various computer vision techniques is the detection of any abnormal activity.

Revised Manuscript Received on August 10, 2019.

Richa Gupta, Graphic Era Deemed to be University, Dehradun, India. E-mail: richa1883gupta@gmail.com amitgupta7920@gmail.com

Dr. Vikas Tripathi, Graphic Era Deemed to be University, Dehradun, India. E-mail: vikastripathi.be@gmail.com

Dr. Devesh Pratap Singh, Graphic Era Deemed to be University, Dehradun, India. E-mail: Devesh.geu@gmail.com

Dr. Bhaskar Pant, Graphic Era Deemed to be University, Dehradun, India. E-mail: pantbhaskar2@gmail.com
Amit Gupta, Graphic Era Hill University, Dehradun, India. E-mail:

It makes the use of various approaches such as image classification, object detection, object tracking which can be helpful in the detection of various activities from the image, motion images or surveillance videos. As the images or videos that are used in computer vision are of various formats and of heavy size, thus simple and traditional methods are incapable for the proper processing of these file. Various big data analytics along with the computer vision concepts can easily overcome this problem. As per the definition of Big Data, it is defined as the various formats of unstructured data which is uncontrollable, and cannot be store and process using basic traditional techniques of database management. In big data the various Vs(Volume, Velocity, Variety, Value and Veracity) needs to be understood very carefully. The emergence and development of certain new approaches and technologies in processing and analysis of big data have given an efficient way to the various computer vision techniques that improves the performance of various computer vision applications. The applications or tools that are used for the detection of abnormal activities can perform better if the analysis of these tools is based on the huge data sets of various formats.

The major challenges that may occur in the area of computer vision are: 1 . As the data grow from the normal capacity to the Big Data the computational overhead increases and that may lead to some performance and efficiency related issues. 2. Quality of the images that are being captured from the surveillance cameras are not at par as they fully dependent on the type of camera being used and the distance between crowd and the cameras. 3. The various methodologies and techniques that are available can work efficiently in less crowded scene but their accuracy degrades as the crowd start becoming dense.

The major challenges in computer vision can be easily handled by using appropriate big data tools and techniques. The table mentioned below shows some tools that can be used to effectively store, process and retrieve data. In the last few years, the number of cameras installed in cities has increased exponentially to monitor various public places like hospitals, schools, roads, air ports, railway stations, malls public places etc., thus the data generated by these cameras is very enormous and is unstructured in nature. Here comes the role of big data techniques and technologies which improves the process of extracting useful information and patterns from the real time images. 


\section{Detecting Abnormal Activities using Computer Vision in Big Data Framework}

Table 1: Areas used for Abnormities Detection

\begin{tabular}{|c|c|c|}
\hline S.No. & Area & $\begin{array}{c}\text { Applications of } \\
\text { Abnormalities } \\
\text { Detection }\end{array}$ \\
\hline 1 & $\begin{array}{l}\text { Law enforcement } \\
\text { and surveillance }\end{array}$ & $\begin{array}{l}\text { Detect traffic rule } \\
\text { violations, suspects } \\
\text { tracking and } \\
\text { investigation }\end{array}$ \\
\hline 2 & Security & $\begin{array}{l}\text { Abnormal crowd } \\
\text { behaviour, fight scene } \\
\text { in public area, } \\
\text { violence detection }\end{array}$ \\
\hline 3 & Transportation & $\begin{array}{l}\text { Detection of road } \\
\text { accidents }\end{array}$ \\
\hline 4 & Social Networking & $\begin{array}{l}\text { Anomalous activity on } \\
\text { social networking like } \\
\text { twitter and facebook }\end{array}$ \\
\hline 5 & Cyber Attack & $\begin{array}{l}\text { Financial Losses and } \\
\text { Cyber Wars }\end{array}$ \\
\hline 6 & Health Care & $\begin{array}{l}\text { Patient monitoring } \\
\text { system }\end{array}$ \\
\hline 7 & Information Security & $\begin{array}{l}\text { Information theft in } \\
\text { healthcare and } \\
\text { financial department }\end{array}$ \\
\hline 8 & Weather Patterns & $\begin{array}{l}\text { Bad Weather } \\
\text { Detection }\end{array}$ \\
\hline 9 & Banking Sectors & $\begin{array}{l}\text { Financial Fraud } \\
\text { Detection }\end{array}$ \\
\hline
\end{tabular}

Table 2: Big Data Tools and Their Applications

\begin{tabular}{|l|l|l|}
\hline S.No. & \multicolumn{1}{|c|}{$\begin{array}{c}\text { Big Data } \\
\text { Components }\end{array}$} & \multicolumn{1}{|c|}{ Applications } \\
\hline 1 & $\begin{array}{l}\text { Hadoop Distributed } \\
\text { File System }\end{array}$ & $\begin{array}{l}\text { Stores and process } \\
\text { distributed data }\end{array}$ \\
\hline 2 & Map Reduce & $\begin{array}{l}\text { Combines the results of } \\
\text { various parallel } \\
\text { executing tasks }\end{array}$ \\
\hline 3 & HBase & $\begin{array}{l}\text { Real Time Database } \\
\text { accessibility and } \\
\text { Processing }\end{array}$ \\
\hline 4 & Hive & $\begin{array}{l}\text { Statistical analysis of } \\
\text { large data ware house } \\
\text { systems }\end{array}$ \\
\hline 5 & Mahout & $\begin{array}{l}\text { Support major ML } \\
\text { algorithms on large } \\
\text { datasets }\end{array}$ \\
\hline 6 & Rapid Miner & $\begin{array}{l}\text { Integrated Environment } \\
\text { for Data Science, ML } \\
\text { and Predictive Analysis }\end{array}$ \\
\hline 7 & Lumify & $\begin{array}{l}\text { Big Data Fusion / } \\
\text { Integration, Analytics } \\
\text { and Visualization }\end{array}$ \\
\hline 8 & Text Analysis & $\begin{array}{l}\text { Technique to extract } \\
\text { some meaningful } \\
\text { knowledge from variety } \\
\text { of Textual data. }\end{array}$ \\
\hline 9 & Audio Analysis & $\begin{array}{l}\text { Audio analysis and } \\
\text { speech recognition }\end{array}$ \\
\hline 10 & Video Analysis & $\begin{array}{l}\text { Surveillance applications } \\
\text { using different videos. }\end{array}$ \\
\hline & & \\
\hline
\end{tabular}

\begin{tabular}{|l|l|l|}
\hline 11 & $\begin{array}{l}\text { Social Networking } \\
\text { Analysis }\end{array}$ & $\begin{array}{l}\text { Techniques to be used } \\
\text { for the analysis social } \\
\text { networking data for the } \\
\text { purpose of sentimental } \\
\text { analysis. }\end{array}$ \\
\hline 12 & Predictive Analysis & $\begin{array}{l}\text { Depicting the future } \\
\text { trends and prediction of } \\
\text { some patterns. }\end{array}$ \\
\hline
\end{tabular}

\section{TECHNIQUES USED IN COMPUTER VISION FOR ABNORMAL ACTIVITY DETECTION}

This section describes the various terminologies and techniques that are mostly used for the detection of any kind of abnormal activity. These techniques are classified as Image Classification, Object Detection, Object Tracking etc. To identify any type of abnormal activity, firstly the image should be classified, and then the particular object is detected either from the image or from the video. Then, if required the object is being tracked. The following section describes these techniques:

Image Classification: Image classification is the concept which is mainly utilized for the categorization of any image. This categorization is done to facilitate the identification of different classes in any object or image. Histogram of oriented gradients (HOG), scale-invariant feature transform (SIFT), and local binary patterns (LBP) are the main methods provided for image classification. SVM is best suited for the image classification because of its capability of training the large datasets and providing accurate and efficient results in comparison of other methods.

Object detection: Object detection refers to the detection of the particular object in any or provided image; it can be utilized for the detection of any abnormal activity. Object detection differs from the image classification as by applying classification and localization to many objects instead of one single object. Out of the various methods available for the object detection, YOLO(you only look once)[1] gives the best results. YOLO is the technique which is efficient even also on large datasets. Object tracking: Object tracking is the process to follow any particular object or group of multiple objects in any scene. The vital applications are abnormal crowd behaviour, violence detection in public area, Tragedy like road accidents, event detection which refers to any abnormal activity over social networking site, cyber attacks etc.

\section{LITERATURE REVIEW}

Traffic accidents are the major cause of injuries and for disabling the people since a long time. If the abnormal behaviour of a vehicle is not detected then the trajedy may happen. To prevent the trajedy the abnormal behaviour of an object is to be detected on time, it enhance the necessity of new technologies are evolved here for processing the live streaming video by which the suspect which can harm the other vehicle can be detected timely. 


\section{IMAGE CLASSIFICATION/OBJECT DETECTION}

The abnormal driving behaviour of vehicle is determined by using the modern method and the detection of any object is done through YOLO [1].This tool has a limitation as it is not efficient where there is sudden acceleration, deceleration, or suddenly change of the lane. Thus, this can be implemented using other techniques and a need for some better technology is emerged.

[5]As in real time scenario large amount of visual data generated, but it is impossible to analyze this data with traditional methods or techniques in computer vision. So, for the analysis and processing of such wide variety of visual data for example like photos or streaming videos and to discover semantic similarities which can be useful for interpretation we require some techniques related to visual big data analysis. The agencies which are responsible for enforcement of Law deployed needs accessibility to a very big network of CCTV cameras those are installed in all the sensitive public areas. For the detection of various traffic violators, road accidents, tracking of various criminals, collecting evidence for any kind abnormal activity etc, the efficient road monitoring system plays an important role. Many different frameworks has been proposed for this purpose.

For detection of the persons who is riding a bike without wearing helmet, many frameworks associated with the visual big data analytics can be applied in real time on the collection of different video retrieved from the city's CCTV cameras. The proposed framework[5] works in two stages, in first stage bike rider is being detected from surveillance videos using the techniques like background subtraction and object segmentation. In the later stage, the method tries to locate the rider's head and then extracts some required features that are useful for the detection whether the biker has helmet or not.

In order to specify the efficiency of various techniques used in the field of computer vision, the performance comparison of the 3 popularly used feature representations techniques, that is, Scale Invariant Feature Transform (SIFT), histogram of oriented gradients (HOG), and local binary patterns (LBP) for classification is used.

\section{OBJECT TRACKING}

There are various different technologies, techniques \& methods that can be used to implement tracking of vehicles and their detection[15].These techniques can be categorized broadly into two approaches. First tracking and the second is non-tracking. In tracking approach the moving object is detected but they can be applied when the very few people are in the scene. The non-tracking approach can be applied even though in the crowd scene.[4] It uses the optical flow or space time Markov Random field(MRF) model. The optical flow is computed for every frame of video. According to the orientation or direction the optical flow is divided into various sections. GMM model is trained for every orientation. A likelihood technique is used for the detection of any anomaly. For parallel computing and stream processing this approach is good.
The very first step in this process is to identify various blobs in the pre-characterized ROI that is Region of Interest. The next phase is to measure and evaluate the different movement vectors among identified blobs. For this, various different methodologies that are used to apply pedestrian detection are described in detailed [15]. The 3 different methods that are used for the objective are as follows: Data Fusion Scheme \& Laser Scanner, Histogram of Oriented Gradients and Linear SVM classifier, Scale invariant feature transformation, HAAR cascade classifier and frontal face algorithm.

\section{ABNORMAL ACTIVITIES DETECTION}

After applying these above techniques the tragedy can be happen. Then trajectory based surveillance are also came into existence. The various related tasks in Computer vision and surveillance are always somehow used for all trajectory analysis. Many works have been proposed for the anomaly detection. We give a systematic review of these works.[2] Here, a study of various applications for visual object trajectories, with reference to the representation of scene, detecting any event, detection of abnormal activities, understanding the object behaviour, video summarization of various videos and synopsis generation are very well described in technique called as multi-object tracking, feature tracking, dense trajectory and optical flow can be used for target the motion in a video. For the detection of various events like walking, fighting and running, some technologies and methodologies such as HMM - Hidden Markov Model, Dirchet process or Bayesian framework are commonly used.

Other than traffic monitoring in smart cities it is also required to detect abnormal crowd activities in public areas. [6] Therefore, for this, the proposed solution which is effectively used for the classification is to use MPEG-7, Particle Filter algorithm and Motion Activity descriptors.

MPEG-7 descriptors belong to Motion Activity descriptor group. Some simple operations and calculations are performed on motion vector components. These calculations are performed on the different compressed images which speed-ups the process of the features selection. Here four descriptors are used. Firstly, the motion intensity, secondly, a histogram of 8-bin for various movement directions, next is the descriptor that is used for describing the size and number of any active objects in any specified scene and lastly descriptor which can be used to describe the ratio between motion intensity in the active region which is largest to the motion intensity in any vedio. Different machine learning algorithms such as $\mathrm{KNN}(\mathrm{K}$ nearest neighbour), Adaboost etc can be used for violence detection.

It basically worked on the frames of the video. It is used to calculate the difference among two consecutive frames for any absolute image[3]. The Lagrangian theory provided the tools for analyzing the term non-local information of motion. So the crowd violence can be detected using this theory.

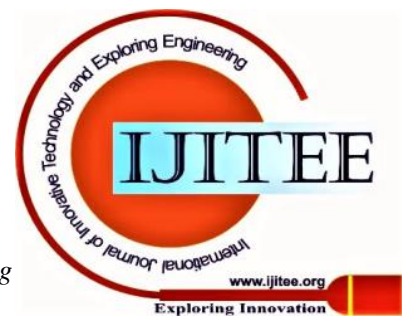




\section{Detecting Abnormal Activities using Computer Vision in Big Data Framework}

SVM (support vector machine) classifier can be utilized for the violence detection in computer vision.

To detect and identify such images in any image sequence that shows an abnormal crowd activity, generally the algorithm namely PF algorithm is used. This PF algorithm can be described as an implementation of recursive Bayes filter technique which is described by Monte Carlo simulations.

The blob sequence optimization is also used to detect various abnormal activities in any video that is used for the purpose of surveillance and then creation of suitable summary videos. Video synopsis is created by the usage of process of optimizing the blob sequence, which basically combines spatial, temporal, size, and motion correlation between different items, this process is done for the extraction of suitable abnormal blob sequences. The various errors that are occurred with respect to occlusion or background interferences can be easily avoided by the use of this process. After which for any long video, a video summary is created by the arrangement of sequences of various abnormal blobs according to their activity types.

In the next few approaches the two important methods: first spatio-temporal descriptors: which is formally a silhouette and optic flow based method and a dense trajectory based technique is used[9]. The technique namely Trajectory shape descriptor is then proposed that can be used to detect abnormalities[9]. Afterwards, SVM technique is used for the classification of any abnormal and normal activities.

For the identification of the abnormal activities, two different approaches can be selected. The first method can be simply defined as a collection various values of optic flow and the characteristics those are based on silhouettes. The other method is based on dense trajectory features those are aligned with the descriptors of HOG, HOF and $\mathrm{MBH}$, which are basically more complex than the previously used techniques.

Within spatio-temporal video volume anomalies can be detected by the analysis of contextual information [8]. Spatio-temporal volumes are built close every pixel, and then the activity pattern codebook is constructed by clustering those volumes. Then, a dictionary is created which is generated using sparse representation. It is used to describe a large spatio-temporal window which is composed of information of the volumes. Furthermore, abnormal events are analyzed by multi-scale analysis. Finally, level of abnormality from any input motion pattern is evaluated by designing the sparse re-construction cost. The above approach is proposed for anomaly detection in crowded scenes.

\section{EVENT DETECTION}

The various and different processes which can be used for the identification of various abnormal or suspicious activity in any the public domain or public platform are termed as anomaly detection[10], which is a well versed mechanism that is used to detect any kind of discrepancies in social networking site by the effective usage of Apache Kafka. The values or information that is received from Kafka producer is than submitted to Hbase database. This data can be used for the purpose of community and anomaly detection afterwards. For the identification of inconsistent nodes, the methods that are mostly used are degree and PII - Political Independence Index metrics. 
Table 3: Comparison of Various Techniques Used for Detection of Abnormal Activity

\begin{tabular}{|c|c|c|c|c|c|c|}
\hline S.NO. & REFERENCES & YEAR & TECNOLOGY USED & APPLICATION & BASE OF TECHNOLOGY & FUTURE WORK \\
\hline 1 & [1] & 2019 & YOLO and Kalman filters & $\begin{array}{l}\text { detecting abnormal vehi- } \\
\text { cle behavior using traffic } \\
\text { camera }\end{array}$ & traffic camera & $\begin{array}{c}\text { Abnormal behaviors such as sudden acceleration } \\
\text { and deceleration, swerving, and sudden lane } \\
\text { change }\end{array}$ \\
\hline 2 & [2] & 2019 & $\begin{array}{l}\text { video summarization and } \\
\text { synopsis generation techniques }\end{array}$ & $\begin{array}{c}\text { surveillance video analysis for } \\
\text { trajedey }\end{array}$ & surveillance cameras & $\begin{array}{l}\text { Deep learning can be used for long duration } \\
\text { tracking }\end{array}$ \\
\hline 3 & [3] & 2019 & $\begin{array}{l}\text { classification method,svm,deep } \\
\text { learning }\end{array}$ & $\begin{array}{c}\text { abnormal and violence } \\
\text { detection }\end{array}$ & $\begin{array}{l}\text { support vector machine } \\
\text { (SVM), } \\
\text { and using deep learning }\end{array}$ & $\begin{array}{l}\text { fast } \mathrm{CNN}, \mathrm{R}-\mathrm{CNN} \text { can be used for detection } \\
\text { violance in crowd scene }\end{array}$ \\
\hline 4 & [4] & 2018 & $\begin{array}{l}\text { Big data environment using } \\
\text { Apache Kafka, Apache Spark } \\
\text { hbase,HDFS }\end{array}$ & $\begin{array}{l}\text { Anaomalous activities on } \\
\text { social networks like } \\
\text { twitter,facebook }\end{array}$ & big data & some machine learning algos can be used \\
\hline 5 & [5] & 2018 & $\begin{array}{c}\text { Real time big data processing } \\
\text { with machine learning algorithm }\end{array}$ & $\begin{array}{c}\text { Networks which leads to cyber } \\
\text { attack, financial loss, } \\
\text { information theft in healthcare, } \\
\text { and cyber war }\end{array}$ & big data & real time analytics using IOT \\
\hline 6 & [6] & 2016 & $\begin{array}{c}\text { OGMM(optical flow as low level } \\
\text { feature and quantizes the } \\
\text { orientation of optical flow) }\end{array}$ & $\begin{array}{l}\text { localize the position of } \\
\text { abnormal with characteristic } \\
\text { of updating incremently }\end{array}$ & GMM, big data & real time data processig needed \\
\hline 7 & [7] & 2016 & Visual big data analytics & $\begin{array}{l}\text { Identify the Violation of traffic } \\
\text { rules }\end{array}$ & video surveillance & $\begin{array}{c}\text { can be extended to detect violation of other rules } \\
\text { and report and detect the number plate of violators } \\
\text { of rules. }\end{array}$ \\
\hline 8 & [8] & 2016 & $\begin{array}{l}\text { MPEG-7 motion activity } \\
\text { descriptors and particle filter } \\
\text { algorithm for classification }\end{array}$ & $\begin{array}{l}\text { to detect the abnormal } \\
\text { behaviour of crowd }\end{array}$ & MPEG & $\begin{array}{l}\text { to modify the algo so that it can localizethe } \\
\text { anomalies in the scene }\end{array}$ \\
\hline 9 & [9] & 2015 & $\begin{array}{c}\text { blob sequence optimization } \\
\text { process which integrates the } \\
\text { spatial,temporal, size and motion } \\
\text { correlation among objects }\end{array}$ & $\begin{array}{l}\text { detect abnormal activities in } \\
\text { surveillance videos }\end{array}$ & video surveillance & $\begin{array}{l}\text { 1.extending to more activitytypes such that the } \\
\text { approach can create summary videos for } \\
\text { any people-interested activity types } \\
\text { 2.combining withtrajectory association methods }\end{array}$ \\
\hline 11 & [10] & 2015 & $\begin{array}{l}\text { GPU based Gaussian Mixture } \\
\text { Model (GMM) }\end{array}$ & $\begin{array}{l}\text { to detect the abnormal } \\
\text { behaviour of crowd }\end{array}$ & GPU with GMM & tracking can be done \\
\hline 12 & [11] & 2014 & $\begin{array}{c}\text { blob analysis, Background } \\
\text { subtraction, Gaussian Mixture } \\
\text { Models } \\
\end{array}$ & $\begin{array}{c}\text { applied to public places where } \\
\text { security is a major issue }\end{array}$ & blob analysis & need technology for all scene and scenario \\
\hline 13 & [12] & 2014 & $\begin{array}{c}\text { Spatio-temporal video,activity } \\
\text { pattern codebook,sparse } \\
\text { representaion }\end{array}$ & anomaly detection in crowd & $\begin{array}{c}\text { weighted } \\
\text { fuzzy c means (WFCM) } \\
\text { algorithm } \\
\end{array}$ & online learning method \\
\hline 14 & [13] & 2014 & $\begin{array}{l}\text { two different spatio-temporal } \\
\text { descriptors, a silhouette and optic } \\
\text { flow based method and a dense } \\
\text { treajectory based method }\end{array}$ & $\begin{array}{c}\text { abnormal activity detection in } \\
\text { crowd }\end{array}$ & DTF and SOF ,SVM & Deep learning can be used \\
\hline 15 & [14] & 2014 & simple moitoring system & patient monitoring system & $\begin{array}{c}\text { intelligent video analytic } \\
\text { system }\end{array}$ & multi modal alanalysis of patient information. \\
\hline
\end{tabular}

\section{CONCLUSION}

The various techniques mainly used for the detection of abnormal activities, violence actions are classification, support vector machine and deep learning. For the identification of the illegal or unlawful activity of any person over the social network platform, the concept of dynamic anomaly detection approach is mostly used. In the detection of such activities the Apache Kafka and Spark streaming of big data framework can be utilized for enhancing its performance. OGMM methodology is widely useful in the detection and localization of exceptional activities of a user.

This paper gives a detailed description of various techniques that can be used in surveillance video analysis. This study depicts the commonly used techniques of big data and computer vision that can enhance the performance of any model which is used for the purpose of detecting abnormal activities.

\section{REFERENCES}

1. Chen Wang, Aibek Musaev, Pezhman Sheinidashtegol, and Travis Atkiso:Towards Detection of Abnormal VehicleBehavior Using Traffic Cameras. In :\# K. Chen et al. (Eds.): BigData 2019, LNCS 11514, pp. 125-136, 2019
2. Sk. Arif Ahmed, Debi Prosad Dogra, Samarjit Kar:Trajectory-Based Surveillance Analysis: A Survey.In:Ieee Transactions On Circuits And Systems For Video Technology, Vol. 29, No. 7, July 2019

3. Muhammad Ramzan , Adnan Abid ,Hikmat Ullah Khan , Shahid Mahmood Awanamina Ismail ,Muzamil Ahmed, Mahwish Ilyas and Ahsan Mahmood. :A Review on State-of-the-Art Violence detection technique. In:Received July 5, 2019, accepted July 18, 2019, date of publication July 30, 2019, date of current version August 19, 2019. Digital Object Identifier 10.1109/ACCESS.2019.2932114

4. Manjunatha H C,Dr. R Mohanasundaram. :BRNADS: Big data RealTime Node Anomaly Detection in Social Networks. Proceedings of the Second International Conference on Inventive Systems and Control (ICISC 2018) IEEE Xplore Compliant - Part Number:CFP18J06-ART, ISBN:978-1-5386-0807-4;

5. Riyaz Ahamed Ariyaluran Habeeb a , Fariza Nasaruddin a, , Abdullah Gani b ,Ibrahim Abaker Targio Hashem b , Ejaz Ahmed c Muhammad Imran. :Real time big data processing for anomaly detection : a survey.In:Received 15 February 2018; Received in revised form 23 August 2018; Accepted 24 August 2018 0268-4012/ (C) 2018 Elsevier Ltd.

6. Feiping Li,Wenming Yang ,Qingmin Liao.: An efficient anomaly detection approach in surveillance video based on oriented GMM .In :978-1-14799-9988-0/16/\$31.00@2016 IEEE

7. Dinesh Singh, C. Vishnu and C. Krishna Mohan. :Visual big data analytics for traffic monitoring in smart city .In:2016 15th IEEE International Conference on Machine Learning and Applications 


\section{Detecting Abnormal Activities using Computer Vision in Big Data Framework}

8. Aukasz KamiĚski, Paweá GardziĚski, Krzysztof Kowalak, Sáawomir Maükowiak.:Unsupervised abnormal crowd activity detection in surveillance system.In:,:978-1-4673-9555-7/16/\$31.00@2016 IEEE.

9. Yihao Zhang, Weiyao Lin, Guangwei Zhang, Chuanfei Luo, Dong Jiang , Chunlian Yao.:A New Approach For Extracting And Summarizing Abnormal Activities Surveillance Videos.

10. Mohammadreza Yavari and Maozhen Li, Maozhen Li and Siguang $\mathrm{Li}$, Man Qi.:parallelizing of abnormal event detection in crowded scene with GPU. In:2015 12th International Conference on Fuzzy Systems and Knowledge Discovery (FSKD)

11. G.Santhiya , K.Sankaragomathi , S.Selvarani , A.Niranjil Kuma. : Abnormal crowd tracking and Motion analysis.In:2014 IEEE International Conference on Advanced Communication Control and Computing Technologies (ICACCCT)

12. Nannan Li, Huiwen Guo, Dan Xu, Xinyu Wu. :Multiscale analysis of contextual information within spatio-temporal video volumes for anomaly detection. In:978-1-4799-5751-4/14/\$31.00 @2014 IEEE

13. K. G. Manosha Chathuramali, Sameera Ramasinghe, Ranga Rodrigo.:Abnormal activity recognition using spatio-temporal feature .In978-1-4799-4598-6/14/\$31.00 \#2014 IEEE

14. Panachit Kittipanya-NgamOng Soh GuatEng HowLung.: Computer Vision Applications for Patients Monitoring System.

15. Shree B V, A, Karthikeyan: Computer Vision based Advanced Driver Assistance System Algorithms with Optimization Techniques International conference on Electronics, Communication and Aerospace Technology (ICECA 2018) IEEE Conference Record \# 42487; IEEE Xplore ISBN:978-1-5386-0965-1

16. Popov S.B: "The Big Data methodology in computer vision systems" (ITNT-2015). 